\title{
The black hole mass of low redshift radiogalaxies
}

\author{
D. Bettoni ${ }^{1}$, R. Falomo ${ }^{1}$, G. Fasano ${ }^{1}$, and F. Govoni ${ }^{2}$ \\ 1 INAF - Osservatorio Astronomico di Padova vicolo Osservatorio 5 Padova, Italy \\ e-mail: falomo@pd.astro.it, fasano@pd.astro.it \\ ${ }^{2}$ Istituto di Radioastronomia di Bologna and Dipartimento di Astronomia, Universitá di Bologna, Italy \\ e-mail: fgovoni@ira.bo.cnr.it
}

Received 9 July 2002 / Accepted 3 December 2002

\begin{abstract}
We make use of two empirical relations between the black hole mass and the global properties (bulge luminosity and stellar velocity dispersion) of nearby elliptical galaxies, to infer the mass of the central black hole $\left(\mathcal{M}_{\mathrm{BH}}\right)$ in low redshift radiogalaxies. Using the most recent determinations of black hole masses for inactive early type galaxies we show that the bulge luminosity and the central velocity dispersion are almost equally correlated (similar scatter) with the central black-hole mass. Applying these relations to two large and homogeneous datasets of radiogalaxies we find that they host black-holes whose mass ranges from $\sim 5 \times 10^{7}$ to $\sim 6 \times 10^{9} M_{\odot}$ (average $<\log \mathcal{M}_{\mathrm{BH}}>\sim 8.9$ ). $\mathcal{M}_{\mathrm{BH}}$ is found to be proportional to the mass of the bulge $\left(\mathcal{M}_{\text {bulge }}\right)$. The distribution of the ratio $\mathcal{M}_{\mathrm{BH}} / \mathcal{M}_{\text {bulge }}$ has a mean value of $8 \times 10^{-4}$ and shows a scatter that is consistent with that expected from the associated errors. At variance with previous claims no significant correlation is instead found between $\mathcal{M}_{\mathrm{BH}}$ (or $\mathcal{M}_{\text {bulge }}$ ) and the radio power at $5 \mathrm{GHz}$.
\end{abstract}

Key words. black hole physics - galaxies: active - galaxies: nuclei - radio continuum: galaxies

\section{Introduction}

There is consensus about the existence of super massive black holes (SBHs) at the center of nearby inactive galaxies as well as in the nuclei of active galaxies and quasars (see e.g. for a recent review Ferrarese 2002). A large body of data, in particular based on high resolution HST observations, is now available (see e.g. Kormendy \& Gebhardt 2001) to support the presence of such massive black holes (BH) using different techniques.

It is believed that SBHs play an important role in the formation and evolution of massive galaxies and also are a key component in the development of the nuclear activity. However in spite of this apparently ubiquitous presence of SBHs in galaxies, our understanding of how the galaxies and their central $\mathrm{BHs}$ are linked in the process of formation of the observed structures is still poor (see Silk \& Rees 1998; Haehlnet \& Kauffmann 2000; Adams et al. 2001).

The most important result obtained from the measured $\mathrm{BH}$ masses in nearby galaxies is the significant correlation between the black hole mass $\left(\mathcal{M}_{\mathrm{BH}}\right)$ and the mass of the bulge component $\left(\mathcal{M}_{\text {bulge }}\right)$ of the host galaxy $\left(\mathcal{M}_{\text {bulge }}\right)$. From the observational point of view this correlation is translated into relationships between $\mathcal{M}_{\mathrm{BH}}$ and bulge luminosity $L_{\text {bulge }}$ (Magorrian et al. 1998; Kormendy \& Gebhardt 2001) and between $\mathcal{M}_{\mathrm{BH}}$ and the stellar velocity dispersion $\sigma$ (Ferrarese \& Merritt 2000; Gebhardt et al. 2000).

Although based on a small number $(\sim 30)$ of nearby galaxies for which direct dynamical measurements of $\mathcal{M}_{\mathrm{BH}}$ have

Send offprint requests to: D. Bettoni, e-mail: bettoni@pd. astro.it been secured, and in spite of their scatter $\left[\sim 0.4\right.$ in $\left.\log \left(\mathcal{M}_{\mathrm{BH}}\right)\right]$, these empirical relationships offer a new tool for evaluating $\mathcal{M}_{\mathrm{BH}}$ in various types of $\mathrm{AGN}$, provided that bulge luminosities and/or velocity dispersions are available (see also McLure \& Dunlop 2002; Falomo et al. 2002).

In this paper we make use of such relationships to investigate the $\mathrm{BH}$ mass distribution of two large and homogeneous datasets of low redshift radiogalaxies (RG) for which we have previously studied the morphological, structural, photometrical and kinematical properties (Fasano et al. 1996; Govoni et al. 2000a, b; Bettoni et al. 2001). The derived BH masses of radiogalaxies are then used to investigate the connections between $\mathcal{M}_{\mathrm{BH}}$, the mass of the galaxy and the radio power.

To this aim we first describe our samples (Sect. 2) and revisit the relations $\mathcal{M}_{\mathrm{BH}}-L_{\text {bulge }}$ and $\mathcal{M}_{\mathrm{BH}}-\sigma$ for nearby earlytype galaxies (Sect. 3). Then we use these relationships to evaluate $\mathcal{M}_{\mathrm{BH}}$ of radio galaxies (Sect. 4) and to study the connections between $\mathcal{M}_{\mathrm{BH}}$ and the mass of the bulge component of the host galaxy and between $\mathcal{M}_{\mathrm{BH}}$ and the radio luminosity. A summary of the main conclusions of this study is reported in Sect. 5. In our analysis we assume $H_{0}=50 \mathrm{~km} \mathrm{~s}^{-1} \mathrm{Mpc}^{-1}$ and $\Omega_{0}=0$.

\section{The samples}

\subsection{The sample of inactive nearby ellipticals}

In order to investigate the relations $\mathcal{M}_{\mathrm{BH}}-L_{\text {bulge }}$ and $\mathcal{M}_{\mathrm{BH}}-\sigma$ of E-type galaxies we have considered a sample of 20 objects 
Table 1. Properties of the nearby inactive galaxy sample.

\begin{tabular}{|c|c|c|c|c|c|c|c|c|c|}
\hline $\begin{array}{l}\text { Object } \\
\text { (1) }\end{array}$ & $\begin{array}{l}\text { Type } \\
\text { (2) }\end{array}$ & $\begin{array}{r}\mathrm{D} \\
\mathrm{Mpc} \\
(3)\end{array}$ & $\begin{array}{l}B_{t} \\
(4)\end{array}$ & $\begin{array}{c}B-R \\
(5)\end{array}$ & $\begin{array}{l}A_{B} \\
\text { (6) }\end{array}$ & $\begin{array}{l}M_{B} \\
\text { (7) }\end{array}$ & $\begin{array}{c}\sigma \\
\left(\mathrm{km} \mathrm{s}^{-1}\right) \\
(8)\end{array}$ & $\begin{array}{c}\log R_{\mathrm{e}} \\
(\mathrm{kpc}) \\
(9)\end{array}$ & $\begin{array}{c}\log \left(\mathcal{M}_{\mathrm{BH}}\right) \\
\mathcal{M}_{\odot} \\
(10)\end{array}$ \\
\hline N221/M32 & $\mathrm{cE} 2$ & 0.81 & 8.52 & 1.53 & 0.55 & -16.02 & 76 & -0.82 & 6.40 \\
\hline N821 & E6 & 24.10 & 11.49 & 1.68 & 0.55 & -20.42 & 196 & 0.72 & 7.57 \\
\hline $\mathrm{N} 2778$ & $\mathrm{E}$ & 22.90 & 13.22 & 1.64 & 0.17 & -18.58 & 171 & 0.26 & 7.15 \\
\hline N3377 & E5+ & 11.20 & 11.07 & 1.37 & 0.21 & -19.18 & 131 & 0.26 & 8.00 \\
\hline N3379 & E1 & 10.60 & 10.32 & 1.57 & 0.21 & -19.81 & 210 & 0.26 & 8.00 \\
\hline N3608 & E2 & 23.00 & 11.64 & 1.51 & 0.13 & -20.17 & 206 & 0.59 & 8.28 \\
\hline N4261 & E2 & 31.60 & 11.27 & 1.61 & 0.13 & -21.23 & 290 & 0.77 & 8.72 \\
\hline N4291 & $\mathrm{E}$ & 26.20 & 12.37 & 1.60 & 0.21 & -19.72 & 269 & 0.27 & 8.49 \\
\hline N4374 & E1 & 18.40 & 10.09 & 1.61 & 0.21 & -21.23 & 286 & 0.69 & 8.63 \\
\hline N4473 & E5 & 15.70 & 11.12 & 1.56 & 0.21 & -19.86 & 188 & 0.28 & 8.04 \\
\hline N4486 & E1 & 16.70 & 9.40 & 1.63 & 0.21 & -21.71 & 345 & 0.92 & 9.48 \\
\hline N4564 & $\mathrm{E}$ & 15.00 & 11.94 & 1.57 & 0.17 & -18.94 & 153 & 0.20 & 7.75 \\
\hline N4649 & E2 & 16.80 & 9.72 & 1.64 & 0.17 & -21.41 & 331 & 0.78 & 9.30 \\
\hline N4697 & E6 & 11.70 & 9.99 & 1.54 & 0.17 & -20.35 & 163 & 0.63 & 8.23 \\
\hline N4742 & $\mathrm{E} 4$ & 15.50 & 11.92 & 1.33 & 0.29 & -19.03 & 93 & -0.05 & 7.15 \\
\hline N5128 & $S 0_{\mathrm{pec}}$ & 4.20 & 7.30 & 1.60 & 0.50 & -20.82 & 145 & $0.82^{a}$ & 8.38 \\
\hline N5845 & $\mathrm{E}^{*}$ & 25.90 & 13.15 & 1.65 & 0.34 & -18.92 & 275 & -0.29 & 8.38 \\
\hline N6251 & $\mathrm{E}$ & 106.00 & 13.18 & 1.60 & 0.42 & -21.95 & 297 & $1.31^{b}$ & 8.72 \\
\hline N7052 & $\mathrm{E}$ & 58.70 & 12.41 & 1.60 & 0.80 & -21.43 & 261 & $0.82^{c}$ & 8.52 \\
\hline I1459 & E3 & 29.20 & 10.88 & 1.59 & 0.07 & -21.45 & 312 & 0.74 & 9.40 \\
\hline
\end{tabular}

${ }^{a}$ From Dufour et al. (1979), ${ }^{b}$ from Owen \& Laing (1989), ${ }^{c}$ from Gonzalez-Serrano et al. (1993).

of E-type morphology (excluding lenticulars) in the Kormendy \& Gebhardt (2001) galaxy list with measured BH masses. The Milky Way was therefore not considered. In Table 1 we report the relevant data for this sample: Cols. 1 and 2 give the name and the morphological type from RC3; Col. 3 the distance, derived from Surface Brightness Fluctuations (SBF, Tonry et al. 2001); Cols. 4-7 report the adopted apparent total $B$ magnitude corrected for extinction, the $B-R$ color, the galactic extinction, derived from the Bell Laboratories Survey of neutral hydrogen (Stark et al. 1992) and the absolute $B$ magnitude respectively. The $B$-band bulge magnitudes were taken from Faber et al. (1997) for all the 20 objects but for NGC 5128, NGC 6251 and NGC 7052 we adopted the magnitudes given in RC3 (de Vaucouleurs et al. 1991). Note that for this sample the bulge magnitude is coincident with the total magnitude of the galaxy since the galaxy luminosity profile is always well represented by an $r^{1 / 4}$ law. The adopted values for the velocity dispersion (from Ferrarese \& Merritt 2000), effective radius $R_{\mathrm{e}}$ (from Faber et al. 1989 and using the distance in Col. 3) and $\mathcal{M}_{\mathrm{BH}}$ are reported in Cols. $8-10$. The latter values are taken from Tremaine et al. (2002) that give the most recent revision of $\mathcal{M}_{\mathrm{BH}}$ for this data set.

\subsection{The radio-galaxies sample}

We have considered the following two samples of radio galaxies:

- Sample A: this consists of 72 radio-galaxies at $z<0.2$ with available values of the absolute magnitude $M_{\mathrm{R}}$, the effective radius $R_{\mathrm{e}}$ and the velocity dispersion $\sigma$. We used these data in a previous work (Bettoni et al. 2001) to study the Fundamental Plane of RGs. In this sample 22 objects were observed by us, 22 galaxies were taken from Smith et al. (1990, SHI90), 16 from Ledlow \& Owen (1995), 7 from Gonzales-Serrano \& Carballo (2000) and 5 from Faber et al. (1989, FA89). In Table 2 we report the relevant data for the radio-galaxies in this sample: in Cols. 1, 2 we give the name and the redshift; in Col. 3 we list the absolute $R$ (Cousins) magnitude to which we have also applied a correction to set the host galaxy luminosity to the present epoch assuming a passive stellar evolution for massive ellipticals (Bressan et al. 1994). Columns 47 report the $\log (\sigma)$, the total and core radio luminosity at $4800 \mathrm{MHz}$ and the reference for these radio data. The velocity dispersion is normalized to a circular aperture of metric radius $1.19 h^{-1} \mathrm{kpc}$ (Jørgensen et al. 1996). This normalization is practically equivalent to that adopted by Ferrarese $\&$ Merritt (2000), who consider $\sigma$ within an aperture radius of $r_{\mathrm{e}} / 8$. The average difference between the two normalizations for our objects is $\leq 5 \mathrm{~km} \mathrm{~s}^{-1}$.

- Sample B: this consists of 79 radiogalaxies at $z<0.1$ for which we secured homogeneous photometric and structural parameters (Fasano et al. 1996; Govoni et al. 2000a, b). These radio galaxies are extracted from two complete surveys of radio sources (Wall \& Peacock 1985: WP, Ekers et al. 1989: EK) according to the specifications given in Fasano et al. (1996), sample B has 22 objects in common 


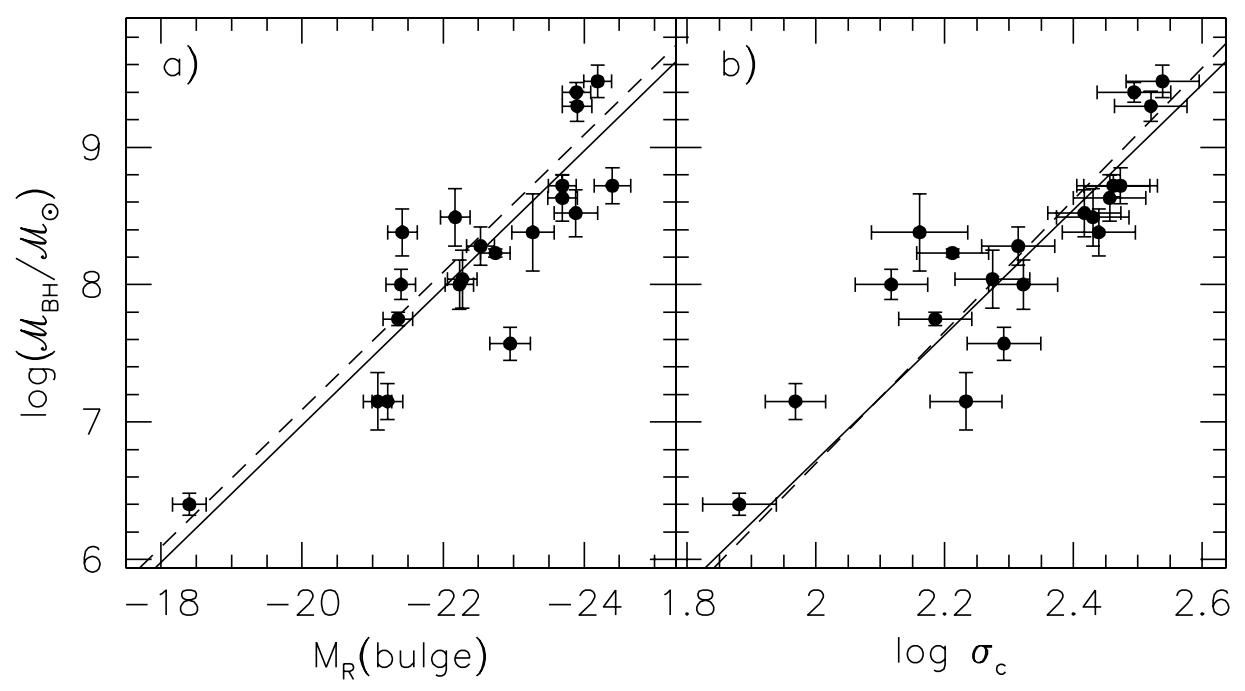

Fig. 1. a) The $\mathcal{M}_{\mathrm{BH}}$ vs. $M_{\text {bulge }}$ relation for the 20 nearby inactive ellipticals (see Table 1). b) Same as a) but for the $\mathcal{M}_{\mathrm{BH}}$ vs. $\log (\sigma)$ relation. The solid lines refer to our linear best fits. The dashed lines represent the relation of McLure \& Dunlop (2002) scaled to our cosmology (panel a)) and that of Ferrarese \& Merritt (2000) (panel b)).

with sample A. In Table 3 we report the relevant data for this sample. In Cols. 1, 2 we give the name and the redshift; in Cols. 3-6 we list the absolute $R$ magnitude, the total and core radio luminosity at $4800 \mathrm{MHz}$ and the reference for the radio data.

\section{The $\mathcal{M}_{\mathrm{BH}}-M_{\mathrm{R}}$ (host) and $\mathcal{M}_{\mathrm{BH}}-\log (\sigma)$ relations for normal, nearby ellipticals}

Using the dataset of nearby inactive ellipticals described in Sect. 2.1 we have derived the best fit of the relations $\log \left(\mathcal{M}_{\mathrm{BH}}\right)-M_{\mathrm{R}}$ (host) and $\log \left(\mathcal{M}_{\mathrm{BH}}\right)-\log (\sigma)$. In order to obtain a relationship between $\mathcal{M}_{\mathrm{BH}}$ and $M_{R}$ usable for $H_{0}=50$ we need to apply a color correction (to convert the $B$ magnitude into the $R$ band) and a term that takes into account the consistency between the adopted distances of nearby galaxies (see Table 1 ) and the chosen value of $H_{0}$. The latter term can be written as $5 \log \left(H_{0}^{\prime} / H_{0}\right)$, where $H_{0}^{\prime}=74 \mathrm{~km} \mathrm{~s}^{-1} \mathrm{Mpc}^{-1}$ (Tonry et al. 2001). The color correction was derived from LEDA (Prugniel $\&$ Maubon 2000) with the exception of NGC 4291 and of the three galaxies for which the $B$ magnitude has been obtained from RC3. For these objects a standard color $(B-R=1.60)$ was assumed (Fukugita et al. 1995).

The data used to fit the two relations $\mathcal{M}_{\mathrm{BH}^{-}} M_{\mathrm{R}}$ (host) and $\mathcal{M}_{\mathrm{BH}}-\log (\sigma)$ are shown in Fig. 1 . The best fit to the data was derived following the procedure outlined in Fasano \& Vio (1988), which takes into account the individual measurement errors in both coordinates and allows us to estimate the residual scatter $s_{\mathrm{r}}$.

The two fitted relations are:

$\log \left(\mathcal{M}_{\mathrm{BH}} / \mathcal{M}_{\odot}\right)=-0.50( \pm 0.06) \times M_{R}-3.00( \pm 1.35)$

$\mathrm{rms}=0.39$

$\log \left(\mathcal{M}_{\mathrm{BH}} / \mathcal{M}_{\odot}\right)=4.55( \pm 0.49) \times \log (\sigma)-2.27( \pm 1.13)$

$\mathrm{rms}=0.41$

where $M_{R}=M_{B}-(B-R)-5 \log \left(H_{0}^{\prime} / H_{0}\right)$. The residual scatters of the two relations are $0.14( \pm 0.05)$ and $0.35( \pm 0.22)$, respectively. In both cases they turn out to be consistent with zero (within $3 \sigma$ ), implying that the observed scatter is almost entirely accounted for by the uncertainty of the measurements. Both relations are in good agreement with the last determinations by McLure \& Dunlop (2002) and Ferrarese (2002), respectively, once the different cosmology is taken into account. However, at variance with previous findings (Ferrarese \& Merritt 2000; Kormendy \& Gebhardt 2001), luminosity and central velocity dispersion appear to be almost equally correlated (very similar scatter) with the black-hole mass. A similar result was found by McLure \& Dunlop (2002) using a slightly different sample of E-type objects. We note, however, that our scatter $(0.39 \mathrm{dex})$ is larger than that $(0.31 \mathrm{dex})$ found by McLure \& Dunlop (2002). Since the data used by these authors are not published it is not possible to further investigate the origin of this difference.

\section{Results}

\subsection{The $\mathrm{BH}$ mass of radio galaxies}

Here we use the relationships $\log \mathcal{M}_{\mathrm{BH}}-M_{\mathrm{R}}$ (bulge) and $\log \mathcal{M}_{\mathrm{BH}}-\log (\sigma)$, derived in Sect. 3, (Eq. (1) and Eq. (2)) to estimate the $\mathrm{BH}$ mass of the two samples (A and $\mathrm{B}$ ) of radio galaxies defined in Sect. 2.2. The $\mathrm{BH}$ masses derived from $\sigma$ and from the $M_{\mathrm{R}}$ are reported in Cols. 8 and 9 of Table 2. The comparison of the two $\mathcal{M}_{\mathrm{BH}}$ determinations for the sample $\mathrm{A}$ is shown in Fig. 2. We find that the mean values of $\log \left(\mathcal{M}_{\mathrm{BH}}\right)$ $(8.66 \pm 0.44$ from Eq. (1) and $8.91 \pm 0.37$ from Eq. (2)) are (1) significantly different. This is mainly attributable to the systematically lower values of $\sigma$ for the 26 radio galaxies in the sub-samples of SHI90 and FA89 (see Fig. 2). To further emphasize this point we plot in Fig 3 the values of $\sigma$ versus $M_{\mathrm{R}}$ (Faber-Jackson relation) for the RGs in sample A and for normal ellipticals from the Jørgensen et al. (1996, JFK96) sample. 
Table 2. The sample A of radio galaxies.

\begin{tabular}{|c|c|c|c|c|c|c|c|c|c|}
\hline Object & (2) & $\begin{array}{l}M_{\mathrm{R}} \\
\text { host } \\
\text { (3) }\end{array}$ & $\begin{array}{c}\log (\sigma) \\
\mathrm{km} \mathrm{s}^{-1} \\
(4)\end{array}$ & $\begin{array}{c}\log \left(L_{48}\right) \\
\text { total } \\
(\mathrm{W} / \mathrm{Hz}) \\
(5)\end{array}$ & $\begin{array}{c}\log \left(L_{48}\right) \\
\text { core } \\
(\mathrm{W} / \mathrm{Hz}) \\
(6)\end{array}$ & Ref. $^{a}$ & $\begin{array}{c}\log \mathcal{M}_{\mathrm{BH}} \\
\text { from } \sigma \\
\mathcal{M}_{\odot} \\
(8)\end{array}$ & $\begin{array}{c}\log \mathcal{M}_{\mathrm{BH}} \\
\text { from } M \text { (bulge) } \\
\mathcal{M}_{\odot} \\
(9)\end{array}$ & $\begin{array}{c}\log \mathcal{M}_{\text {bulge }} \\
\mathcal{M}_{\odot} \\
(10)\end{array}$ \\
\hline 0055-016 & 0.045 & -24.08 & 2.48 & 25.30 & 23.93 & WP & 9.00 & 8.99 & 12.10 \\
\hline $0131-367$ & 0.030 & -24.10 & 2.40 & 25.21 & 23.18 & WP & 8.64 & 9.01 & 11.92 \\
\hline $0257-398$ & 0.066 & -23.73 & 2.34 & 24.94 & $<22.9$ & EK & 8.79 & 8.80 & 11.75 \\
\hline $0312-343$ & 0.067 & -24.02 & 2.41 & 24.66 & 23.71 & EK & 8.68 & 8.95 & 12.18 \\
\hline $0325+023$ & 0.030 & -23.41 & 2.34 & 24.90 & 23.80 & WP & 8.37 & 8.66 & 11.93 \\
\hline 0449-175 & 0.031 & -23.97 & 2.20 & 24.12 & 22.63 & EK & 8.04 & 8.94 & 11.73 \\
\hline 0546-329 & 0.037 & -24.49 & 2.59 & 24.13 & 23.29 & EK & 9.49 & 9.20 & 12.46 \\
\hline 0548-317 & 0.034 & -23.35 & 2.09 & 24.35 & $<22.49$ & EK & 9.31 & 8.63 & 12.12 \\
\hline 0718-340 & 0.029 & -24.20 & 2.52 & 24.55 & 23.05 & EK & 9.32 & 9.06 & 12.20 \\
\hline 0915-118 & 0.054 & -24.46 & 2.44 & 26.26 & 24.46 & WP & 8.85 & 9.17 & 12.26 \\
\hline 0940-304 & 0.038 & -23.83 & 2.59 & 24.09 & 23.48 & EK & 9.50 & 8.87 & 12.11 \\
\hline $1043-290$ & 0.060 & -24.71 & 2.36 & 24.66 & 23.96 & EK & 8.98 & 9.30 & 12.57 \\
\hline $1107-372$ & 0.010 & -24.61 & 2.47 & 23.07 & 21.64 & EK & 8.96 & 9.27 & 12.07 \\
\hline $1123-351$ & 0.032 & -24.67 & 2.65 & 24.56 & 23.50 & EK & 9.75 & 9.29 & 12.72 \\
\hline $1258-321$ & 0.017 & -24.48 & 2.42 & 24.00 & 23.10 & EK & 8.75 & 9.20 & 12.12 \\
\hline $1333-337$ & 0.013 & -24.55 & 2.46 & 24.67 & 23.34 & WP & 9.04 & 9.24 & 12.17 \\
\hline $1400-337$ & 0.014 & -25.02 & 2.49 & 23.52 & 22.11 & EK & 9.05 & 9.47 & 12.42 \\
\hline $1404-267$ & 0.022 & -24.00 & 2.47 & 23.93 & 23.78 & EK & 8.98 & 8.96 & 12.19 \\
\hline $1514+072$ & 0.034 & -24.86 & 2.43 & 24.70 & 24.31 & WP & 8.57 & 9.38 & 12.36 \\
\hline $1521-300$ & 0.020 & -22.01 & 2.22 & 23.83 & 23.43 & EK & 7.82 & 7.97 & 11.04 \\
\hline $2236-176$ & 0.070 & -24.88 & 2.39 & 25.08 & 23.35 & EK & 8.58 & 9.37 & 12.32 \\
\hline $2333-327$ & 0.052 & -23.77 & 2.43 & 24.20 & 23.17 & EK & 8.77 & 8.83 & 11.90 \\
\hline \multicolumn{10}{|c|}{ Smith et al. (1990) } \\
\hline $3 \mathrm{C} 29$ & 0.044 & -24.18 & 2.32 & 25.30 & 23.92 & WP & 8.27 & 9.04 & 11.88 \\
\hline $3 \mathrm{C} 31$ & 0.016 & -23.69 & 2.40 & 24.41 & - & KK & 8.62 & 8.81 & 12.04 \\
\hline $3 \mathrm{C} 33$ & 0.058 & -23.47 & 2.36 & 25.91 & - & KK & 8.48 & 8.68 & 11.86 \\
\hline $3 \mathrm{C} 62$ & 0.146 & -23.82 & 2.44 & 26.29 & 24.42 & WP & 8.81 & 8.81 & 12.20 \\
\hline $3 \mathrm{C} 76.1$ & 0.032 & -23.21 & 2.30 & 24.82 & - & KK & 8.20 & 8.56 & 11.45 \\
\hline $3 \mathrm{C} 78$ & 0.028 & -24.60 & 2.42 & 25.13 & 24.55 & WP & 8.73 & 9.26 & 11.99 \\
\hline $3 \mathrm{C} 84$ & 0.017 & -24.37 & 2.40 & 25.81 & - & $\mathrm{BB}$ & 8.61 & 9.15 & 12.08 \\
\hline $3 \mathrm{C} 88$ & 0.030 & -23.49 & 2.28 & 24.91 & 23.81 & WP & 8.08 & 8.70 & 11.99 \\
\hline $3 \mathrm{C} 89$ & 0.138 & -24.10 & 2.40 & 25.87 & - & G1 & 8.64 & 8.95 & 12.47 \\
\hline 3C98 & 0.030 & -22.83 & 2.24 & 25.32 & - & KK & 7.91 & 8.37 & 11.56 \\
\hline $3 \mathrm{C} 120$ & 0.033 & -23.78 & 2.30 & 25.63 & 25.24 & WP & 8.20 & 8.84 & 11.81 \\
\hline $3 \mathrm{C} 192$ & 0.061 & -23.21 & 2.28 & 25.54 & - & KK & 8.12 & 8.55 & 11.57 \\
\hline 3C196.1 & 0.199 & -24.66 & 2.32 & 26.02 & - & G1 & 8.29 & 9.21 & 12.17 \\
\hline $3 \mathrm{C} 223$ & 0.138 & -23.49 & 2.31 & 26.07 & - & KK & 8.22 & 8.65 & 11.87 \\
\hline $3 C 293$ & 0.046 & -23.95 & 2.27 & 25.24 & - & KK & 8.04 & 8.92 & 11.48 \\
\hline $3 \mathrm{C} 305$ & 0.042 & -24.16 & 2.25 & 24.91 & - & BB & 7.97 & 9.03 & 11.31 \\
\hline
\end{tabular}

It is clearly apparent that RGs belonging to the SHI90 and FA89 samples deviate systematically from the overall relation derived from fitting the data for the RGs in sample A (excluding the SHI90 and FA89 galaxies) and for normal ellipticals from JFK96 $\left(\log \sigma=-0.375-0.118 \times M_{\mathrm{R}}\right)$. Note also that the galaxies in these datasets do not well agree with the other RGs of sample A in the Fundamental Plane (Bettoni et al. 2001). Therefore, in the following analysis, we exclude the measurements of these 26 RGs. If we now consider the remaining 45 RGs in sample $A$ (hereafter reduced sample A) we obtain: $<\log \left(\mathcal{M}_{\mathrm{BH}}\right)>_{\sigma}=8.81 \pm 0.41$ and $<\log \mathcal{M}_{\mathrm{BH}}>_{M_{\mathrm{R}}}=$ $8.91 \pm 0.40$. For sample $\mathrm{B} \mathcal{M}_{\mathrm{BH}}$ was derived only from $M_{\mathrm{R}}$
(Eq. (1)). These values are given in Table 3. The mean value of $\mathcal{M}_{\mathrm{BH}}<\log \mathcal{M}_{\mathrm{BH}}>_{M_{\mathrm{R}}}=8.94 \pm 0.34$ is in good agreement with those obtained for the reduced sample A. In Fig. 4 the distributions of $\mathcal{M}_{\mathrm{BH}}$ for both samples are compared.

\subsection{The $\mathcal{M}_{B H}-\mathcal{M}_{\text {bulge }}$ relation}

McLure \& Dunlop (2002) and Laor (2001) investigated the relation $\mathcal{M}_{\mathrm{BH}} \propto \mathcal{M}_{\text {bulge }}^{\eta}$ for Seyfert galaxies and powerful QSO in order to test its linearity (which means $\eta=1$ ). In both papers the above relation is derived from $L_{\text {bulge }}$ under the assumption of a mass-to-light ratio having the form $\mathcal{M} / L \propto L^{\Gamma}$ but 
Table 2. continued.

\begin{tabular}{|c|c|c|c|c|c|c|c|c|c|}
\hline Object & $(2)$ & $\begin{array}{l}M_{\mathrm{R}} \\
\text { host } \\
\text { (3) }\end{array}$ & $\begin{array}{l}\log (\sigma) \\
\mathrm{km} \mathrm{s}^{-1} \\
(4)\end{array}$ & $\begin{array}{c}\log \left(L_{48}\right) \\
\text { total } \\
(\mathrm{W} / \mathrm{Hz}) \\
(5)\end{array}$ & $\begin{array}{c}\log \left(L_{48}\right) \\
\text { core } \\
(\mathrm{W} / \mathrm{Hz}) \\
(6)\end{array}$ & Ref. $^{a}$ & $\begin{array}{c}\log \mathcal{M}_{\mathrm{BH}} \\
\text { from } \sigma \\
\mathcal{M}_{\odot} \\
(8)\end{array}$ & $\begin{array}{c}\log \mathcal{M}_{\mathrm{BH}} \\
\text { from } M \text { (bulge) } \\
\mathcal{M}_{\odot} \\
(9)\end{array}$ & $\begin{array}{c}\log \mathcal{M}_{\text {bulge }} \\
\mathcal{M}_{\odot} \\
(10)\end{array}$ \\
\hline 3C338 & 0.031 & -24.9 & 2.46 & 24.29 & - & $\mathrm{BB}$ & 8.93 & 9.40 & 12.55 \\
\hline $3 \mathrm{C} 388$ & 0.090 & -24.92 & 2.56 & 25.83 & - & KK & 9.39 & 9.39 & 12.83 \\
\hline PKS0634-206 & 0.056 & -24.26 & 2.29 & 25.7 & 23.23 & WP & 8.15 & 9.07 & 11.52 \\
\hline PKS2322-122 & 0.081 & -24.33 & 2.35 & 25.11 & - & G2 & 8.42 & 9.10 & 12.24 \\
\hline $3 \mathrm{C} 444$ & 0.152 & -24.77 & 2.19 & 26.4 & $<23.37$ & WP & 7.69 & 9.28 & 12.21 \\
\hline $3 \mathrm{C} 449$ & 0.016 & -22.86 & 2.35 & 24.25 & - & KK & 8.42 & 8.39 & 12.19 \\
\hline \multicolumn{10}{|c|}{ Gonzalez-Serrano \& Carballo (2000) } \\
\hline NGC507 & 0.015 & -23.94 & 2.52 & - & - & - & 9.18 & 8.93 & 12.10 \\
\hline NGC703 & 0.015 & -23.98 & 2.38 & - & - & - & 8.57 & 8.95 & 12.21 \\
\hline $\operatorname{gin} 116$ & 0.033 & -24.01 & 2.45 & 23.76 & - & BB & 8.90 & 8.96 & 12.00 \\
\hline NGC4869 & 0.023 & -23.03 & 2.30 & 23.38 & - & BB & 8.19 & 8.48 & 11.45 \\
\hline NGC4874 & 0.025 & -24.89 & 2.42 & 23.33 & - & $\mathrm{BB}$ & 8.76 & 9.40 & 12.40 \\
\hline NGC6086 & 0.032 & -24.04 & 2.51 & - & - & - & 9.14 & 8.98 & 12.26 \\
\hline NGC6137 & 0.031 & -25.57 & 2.47 & 23.87 & - & $\mathrm{BB}$ & 8.97 & 9.74 & 12.51 \\
\hline \multicolumn{10}{|c|}{ Ledlow \& Owen (1995) } \\
\hline 0039-095B & 0.055 & -22.69 & 2.45 & 23.81 & - & G1 & 8.86 & 8.29 & 11.32 \\
\hline 0053-015 & 0.038 & -24.03 & 2.47 & 24.66 & - & G1 & 8.98 & 8.97 & 12.20 \\
\hline 0053-016 & 0.043 & -23.33 & 2.40 & 24.57 & - & G1 & 8.63 & 8.62 & 11.72 \\
\hline $0110+152$ & 0.044 & -23.87 & 2.29 & 24.36 & - & BB & 8.16 & 8.88 & 11.90 \\
\hline $0112-000$ & 0.045 & -22.94 & 2.40 & 23.58 & - & BB & 8.65 & 8.42 & 11.67 \\
\hline $0122+084$ & 0.049 & -24.71 & 2.56 & - & - & - & 9.39 & 9.30 & 12.69 \\
\hline $0147+360$ & 0.017 & -23.00 & 2.38 & - & - & - & 8.58 & 8.46 & 11.43 \\
\hline 0306-237 & 0.067 & -23.44 & 2.40 & - & - & - & 8.63 & 8.66 & 11.67 \\
\hline 0431-133 & 0.033 & -24.46 & 2.43 & 23.32 & - & G2 & 8.78 & 9.18 & 12.31 \\
\hline 0431-134 & 0.035 & -22.75 & 2.35 & 24.05 & - & G2 & 8.40 & 8.33 & 11.47 \\
\hline $1510+076$ & 0.043 & -22.39 & 2.53 & - & - & - & 9.22 & 8.15 & 11.57 \\
\hline $1520+087$ & 0.034 & -24.20 & 2.34 & - & - & - & 8.38 & 9.05 & 12.25 \\
\hline $1602+178 \mathrm{~A}$ & 0.031 & -22.80 & 2.33 & - & - & - & 8.32 & 8.36 & 11.57 \\
\hline $1610+296$ & 0.032 & -23.88 & 2.51 & - & - & - & 9.14 & 8.90 & 11.90 \\
\hline $2322+143 a$ & 0.045 & -22.36 & 2.31 & 23.99 & - & $\mathrm{BB}$ & 8.24 & 8.13 & 11.24 \\
\hline $2335+267$ & 0.030 & -24.60 & 2.54 & 25.13 & - & $\mathrm{BB}$ & 9.28 & 9.26 & 12.49 \\
\hline \multicolumn{10}{|c|}{ Faber et al. (1989) } \\
\hline NGC315 & 0.016 & -25.13 & 2.49 & 24.15 & - & $\mathrm{BB}$ & 9.31 & 9.53 & 12.57 \\
\hline NGC741 & 0.018 & -24.73 & 2.45 & 23.65 & - & G1 & 8.86 & 9.33 & 12.37 \\
\hline NGC4839 & 0.026 & -23.82 & 2.39 & 22.94 & - & $\mathrm{BB}$ & 8.71 & 8.87 & 12.20 \\
\hline NGC7626 & 0.010 & -22.98 & 2.51 & 23.14 & - & G1 & 8.51 & 8.46 & 11.85 \\
\hline $3 \mathrm{C} 40$ & 0.017 & -23.66 & 2.23 & 24.43 & 23.15 & WP & 7.89 & 8.79 & 11.17 \\
\hline
\end{tabular}

${ }^{a}$ KK - Küehr et al. (1981); G1 - Griffith et al. (1995); G2 - Griffith et al. (1994); BB - Becker et al. (1991).

they reached different conclusions. Laor (2001) claimed that the relation $\mathcal{M}_{\mathrm{BH}}-\mathcal{M}_{\text {bulge }}$ is not linear, while this was not confirmed by McLure \& Dunlop (2002). However, the latter authors showed also that the Laor data may be consistent with linearity if one adopts $\Gamma=0.31$ (Jørgensen et al. 1996) instead of $\Gamma=0.18$ (used by Laor).

Here we investigate the $\mathcal{M}_{\mathrm{BH}} \propto \mathcal{M}_{\text {bulge }}^{\eta}$ relation for our samples of RGs. We have computed the two involved quantities $\left(\mathcal{M}_{\mathrm{BH}}\right.$ and $\left.\mathcal{M}_{\text {bulge }}\right)$ from $\sigma, M_{\mathrm{R}}$ and the effective radius of the galaxy $\left(R_{\mathrm{e}}\right)$ avoiding possible spurious effects introduced in the analysis by the use of the same variable to derive the two masses. We first derive $\mathcal{M}_{\mathrm{BH}}$ from $M_{\mathrm{R}}$ (Eq. (1)) and $\mathcal{M}_{\text {bulge }}$ from $R_{\mathrm{e}}$ and $\sigma$ using the formula $\mathcal{M}_{\text {bulge }}=5 G^{-1} \sigma^{2} R_{\mathrm{e}}$, proposed by Bender et al. (1992). These two determinations are reported in Cols. 9 and 10 of Table 2. Figure 5 shows the linear fit to the $\mathcal{M}_{\mathrm{BH}}-\mathcal{M}_{\text {bulge }}$ relation for objects in the reduced sample A and for normal ellipticals (JFK96), together with the data relative to the sample of inactive galaxies for comparison. We find:

$\log \left(\mathcal{M}_{\mathrm{BH}}\right)=0.96( \pm 0.03) \times \log \left(\mathcal{M}_{\text {bulge }}\right)-2.56( \pm 0.34)$.

This relation has $r m s=0.14$, while the residual scatter (i.e. the scatter not accounted for by the errors) is $s_{\mathrm{r}}=0.03 \pm$ 0.012 , which is consistent with zero. The slope $(\eta=0.96)$ is 
Table 3. The sample B of radio galaxies.

\begin{tabular}{|c|c|c|c|c|c|c|}
\hline Object & (2) & $\begin{array}{l}M_{\mathrm{R}} \\
\text { host } \\
\text { (3) }\end{array}$ & $\begin{array}{c}\log \left(L_{48}\right) \\
\text { total } \\
(\mathrm{W} / \mathrm{Hz}) \\
(4)\end{array}$ & $\begin{array}{c}\log \left(L_{48}\right) \\
\text { core } \\
(\mathrm{W} / \mathrm{Hz}) \\
(5)\end{array}$ & $\begin{array}{l}\text { Ref. } \\
\text { (6) }\end{array}$ & $\begin{array}{c}\log \mathcal{M}_{\mathrm{BH}} \\
\text { from } M_{\text {(bulge) }} \\
\mathcal{M}_{\odot} \\
(7)\end{array}$ \\
\hline 0005-199 & 0.121 & -24.46 & 25.25 & 24.00 & EK & 9.20 \\
\hline 0013-316 & 0.107 & -24.51 & 24.85 & 23.44 & EK & 9.23 \\
\hline $0023-333$ & 0.05 & -24.57 & 24.67 & 23.05 & EK & 9.26 \\
\hline 0034-014 & 0.073 & -23.67 & 25.60 & 24.87 & WP & 8.81 \\
\hline 0055-016 & 0.045 & -24.10 & 25.30 & 23.93 & WP & 9.02 \\
\hline 0123-016 & 0.018 & -24.02 & 24.43 & 23.15 & WP & 8.98 \\
\hline $0131-367$ & 0.03 & -24.13 & 25.21 & 23.18 & WP & 9.04 \\
\hline 0229-208 & 0.089 & -24.10 & 25.25 & 24.54 & EK & 9.02 \\
\hline 0247-207 & 0.087 & -25.21 & 25.03 & 23.91 & EK & 9.58 \\
\hline $0255+058$ & 0.023 & -22.95 & 24.66 & 22.96 & WP & 8.45 \\
\hline 0257-398 & 0.066 & -23.67 & 24.94 & $<22.9$ & EK & 8.81 \\
\hline 0307-305 & 0.066 & -23.24 & 24.89 & 22.78 & EK & 8.59 \\
\hline $0312-343$ & 0.067 & -23.99 & 24.66 & 23.71 & EK & 8.97 \\
\hline $0325+023$ & 0.03 & -23.61 & 24.90 & 23.8 & WP & 8.78 \\
\hline $0332-391$ & 0.063 & -23.82 & 25.13 & 23.37 & EK & 8.88 \\
\hline 0344-345 & 0.053 & -22.79 & 25.25 & 23.71 & EK & 8.37 \\
\hline $0349-278$ & 0.066 & -22.94 & 25.65 & 23.51 & EK & 8.44 \\
\hline $0427-539$ & 0.038 & -24.13 & 25.34 & 23.57 & WP & 9.04 \\
\hline $0430+052$ & 0.033 & -23.11 & 25.62 & 25.22 & WP & 8.53 \\
\hline $0434-225$ & 0.069 & -24.80 & 24.93 & 23.30 & EK & 9.37 \\
\hline 0446-206 & 0.073 & -23.47 & 24.73 & 23.35 & EK & 8.71 \\
\hline 0449-175 & 0.031 & -23.88 & 24.12 & 22.63 & EK & 8.91 \\
\hline $0452-190$ & 0.039 & -23.88 & 24.11 & 23.23 & EK & 8.91 \\
\hline 0453-206 & 0.035 & -24.07 & 25.00 & 23.34 & WP & 9.01 \\
\hline 0511-305 & 0.058 & -23.10 & 25.20 & 23.19 & EK & 8.52 \\
\hline 0533-377 & 0.096 & -24.32 & 24.84 & 23.75 & EK & 9.13 \\
\hline 0546-329 & 0.037 & -24.42 & 24.13 & 23.29 & EK & 9.18 \\
\hline 0548-317 & 0.034 & -23.12 & 24.35 & $<22.49$ & EK & 8.53 \\
\hline $0620-526$ & 0.051 & -24.92 & 25.17 & 24.49 & WP & 9.43 \\
\hline $0625-354$ & 0.055 & -24.43 & 25.46 & 24.92 & WP & 9.19 \\
\hline $0625-536$ & 0.054 & -25.20 & 25.39 & 23.75 & WP & 9.57 \\
\hline 0634-205 & 0.056 & -23.78 & 25.70 & 23.23 & EK & 8.86 \\
\hline 0712-349 & 0.044 & -24.16 & 24.17 & 23.34 & EK & 9.05 \\
\hline 0718-340 & 0.029 & -24.07 & 24.55 & 23.05 & EK & 9.01 \\
\hline 0806-103 & 0.11 & -23.96 & 25.98 & 24.50 & WP & 8.95 \\
\hline 0915-118 & 0.054 & -24.36 & 26.26 & 24.46 & WP & 9.15 \\
\hline 0940-304 & 0.038 & -23.70 & 24.09 & 23.48 & EK & 8.82 \\
\hline $0945+076$ & 0.086 & -23.11 & 25.96 & 24.05 & WP & 8.53 \\
\hline $1002-320$ & 0.089 & -24.20 & 24.93 & $<23.27$ & EK & 9.07 \\
\hline
\end{tabular}

practically coincident with an almost perfect linearity $(\eta=1$; see the dotted line in Fig. 5). This suggests that a fundamental link is present in the combined formation of BHs and spheroids that holds for various types of active and inactive galaxies. We also note that, given the coefficients of the fundamental plane (FP), the linearity of the $\mathcal{M}_{\mathrm{BH}}-\mathcal{M}_{\text {bulge }}$ relation turns out to be directly linked to the particular value of the slope $(\mathrm{A})$ of the relation $\left(\log \mathcal{M}_{\mathrm{BH}}=A \times M_{\mathrm{R}}+B\right)$. In fact the exponent $\eta$ of the $M_{\mathrm{BH}}-M_{\text {bulge }}$ relation can be written in the form: $\eta=-A \alpha / 2 \beta$, where $\alpha$ and $\beta$ are the coefficients of the FP. Using $\alpha=1.242$, $\beta=0.33$ (Bettoni et al. 2001) and $A=-0.5$ it follows that, $\eta=0.94$. Vice versa, if perfect linearity is assumed using the same $\alpha, \beta$ one obtains $A=-0.53$.

The distribution of the black-hole to bulge mass ratio is shown in Fig. 6 (panel a). The average value of the mass ratio is $\left\langle\log \left(\mathcal{M}_{\mathrm{BH}} / \mathcal{M}_{\text {bulge }}\right)>=-3.11\right.$, with $\mathrm{rms} \sim 0.17$. The dispersion expected from just the uncertainties on the measurements $(\sim 0.26)$ is consistent with that observed, suggesting that the intrinsic variance of the ratio $\mathcal{M}_{\mathrm{BH}} / \mathcal{M}_{\text {bulge }}$ is very small.

Alternatively we can derive the $\mathcal{M}_{\mathrm{BH}} / \mathcal{M}_{\text {bulge }}$ ratio using $\mathcal{M}_{\mathrm{BH}}$ derived from $\sigma$ and assuming $\mathcal{M}_{\text {bulge }}=0.0021 L^{1.30}$ (the coefficients are derived by fitting the $\mathcal{M}-L$ relation, in the 
Table 3. continued.

\begin{tabular}{|c|c|c|c|c|c|c|}
\hline Object & (2) & $\begin{array}{l}M_{\mathrm{R}} \\
\text { host } \\
\text { (3) }\end{array}$ & $\begin{array}{c}\log \left(L_{48}\right) \\
\text { total } \\
(\mathrm{W} / \mathrm{Hz}) \\
(4)\end{array}$ & $\begin{array}{c}\log \left(L_{48}\right) \\
\text { core } \\
(\mathrm{W} / \mathrm{Hz}) \\
(5)\end{array}$ & $\begin{array}{l}\text { Ref. } \\
\text { (6) }\end{array}$ & $\begin{array}{c}\log \mathcal{M}_{\mathrm{BH}} \\
M_{\text {(bulge) }} \\
\mathcal{M}_{\odot} \\
(7)\end{array}$ \\
\hline $1043-290$ & 0.06 & -24.62 & 24.66 & 23.96 & EK & 9.28 \\
\hline $1053-282$ & 0.061 & -23.99 & 25.18 & 24.29 & EK & 8.97 \\
\hline $1056-360$ & 0.07 & -23.54 & 25.06 & 24.05 & EK & 8.74 \\
\hline $1107-372$ & 0.01 & -24.37 & 23.07 & 21.64 & EK & 9.16 \\
\hline $1123-351$ & 0.032 & -24.53 & 24.56 & 23.50 & EK & 9.23 \\
\hline $1251-122$ & 0.015 & -24.16 & 24.39 & 22.94 & WP & 9.05 \\
\hline $1251-289$ & 0.057 & -25.31 & 24.55 & $<23.17$ & EK & 9.63 \\
\hline $1257-253$ & 0.065 & -24.09 & 24.72 & 23.49 & EK & 9.02 \\
\hline $1258-321$ & 0.017 & -24.35 & 24.00 & 23.10 & EK & 9.15 \\
\hline $1318-434$ & 0.011 & -24.13 & 23.96 & 23.49 & WP & 9.04 \\
\hline $1323-271$ & 0.044 & -24.00 & 24.64 & 23.12 & EK & 8.97 \\
\hline $1333-337$ & 0.013 & -24.50 & 24.67 & 23.34 & WP & 9.22 \\
\hline $1344-241$ & 0.02 & -23.05 & 23.55 & $<21.85$ & EK & 8.50 \\
\hline $1354-251$ & 0.038 & -23.46 & 24.29 & 22.51 & EK & 8.70 \\
\hline $1400-337$ & 0.014 & -24.90 & 23.52 & 22.11 & EK & 9.42 \\
\hline $1404-267$ & 0.022 & -23.91 & 23.93 & 23.78 & EK & 8.93 \\
\hline $1514+072$ & 0.034 & -24.77 & 24.70 & 24.31 & WP & 9.36 \\
\hline $1521-300$ & 0.02 & -21.78 & 23.83 & 23.43 & EK & 7.86 \\
\hline $1637-771$ & 0.041 & -23.41 & 25.29 & 24.14 & WP & 8.68 \\
\hline 1717-009 & 0.031 & -22.49 & 25.94 & 23.70 & WP & 8.22 \\
\hline $1733-565$ & 0.098 & -23.68 & 26.19 & 25.49 & WP & 8.81 \\
\hline $1928-340$ & 0.098 & -24.63 & 24.94 & 23.98 & EK & 9.29 \\
\hline $1929-397$ & 0.073 & -25.04 & 25.39 & 23.54 & EK & 9.49 \\
\hline $1949+023$ & 0.059 & -23.69 & 25.58 & 23.2 & WP & 8.82 \\
\hline $1954-552$ & 0.058 & -23.33 & 25.55 & 23.89 & WP & 8.64 \\
\hline 2013-308 & 0.088 & -24.69 & 25.01 & 23.56 & EK & 9.32 \\
\hline $2031-359$ & 0.088 & -24.54 & 25.33 & 23.64 & EK & 9.24 \\
\hline $2040-267$ & 0.041 & -24.09 & 24.83 & 23.37 & EK & 9.02 \\
\hline $2058-282$ & 0.039 & -24.24 & 25.13 & 23.63 & WP & 9.09 \\
\hline $2059-311$ & 0.039 & -24.23 & 24.13 & 23.09 & EK & 9.09 \\
\hline $2104-256$ & 0.038 & -24.20 & 25.46 & 23.59 & WP & 9.07 \\
\hline $2128-388$ & 0.018 & -23.20 & 23.76 & 22.46 & EK & 8.57 \\
\hline $2158-380$ & 0.034 & -23.16 & 24.47 & $<22.4$ & EK & 8.55 \\
\hline $2209-255$ & 0.063 & -24.23 & 24.67 & 23.95 & EK & 9.08 \\
\hline $2221-023$ & 0.057 & -22.36 & 25.53 & 24.10 & WP & 8.15 \\
\hline $2225-308$ & 0.056 & -22.69 & 24.69 & 23.63 & EK & 8.32 \\
\hline $2236-176$ & 0.07 & -24.70 & 25.08 & 23.35 & EK & 9.32 \\
\hline $2333-327$ & 0.052 & -23.77 & 24.20 & 23.17 & EK & 8.86 \\
\hline $2350-375$ & 0.116 & -24.03 & 25.13 & $<23.41$ & EK & 8.99 \\
\hline $2353-184$ & 0.073 & -23.92 & 24.88 & $<23.39$ & EK & 8.93 \\
\hline
\end{tabular}

$R$ band, for our RG data together with the ellipticals in JFK96). In this case we obtain $<\log \left(\mathcal{M}_{\mathrm{BH}} / \mathcal{M}_{\text {bulge }}\right)>=-3.16$ with a dispersion of 0.43 (Fig. 6b). These are in good agreement with those derived for the inactive galaxy sample: -3.07 ( $\mathrm{rms}=$ 0.19 ) and $-3.05(\mathrm{rms}=0.50)$ for the two procedures respectively. They are also similar to previous determinations by Merritt \& Ferrarese (2001) who found $-2.90 \pm 0.45$ using the Magorrian et al. (1998) galaxy sample and the $\mathcal{M}_{\mathrm{BH}^{-}} \sigma$ relation, and by McLure \& Dunlop (2002) who report $-2.87 \pm 0.47$ from their study of the host galaxies of powerful quasars and use virial black-hole masses.

\subsection{Relationship between $\mathcal{M}_{B H}$ and radio emission}

Based on a small number of nearby galaxies with known BH masses it was suggested by Franceschini et al. (1998) that $\mathcal{M}_{\mathrm{BH}}$ scales with the total radio luminosity $L_{\text {radio }}$ at $5 \mathrm{GHz}$ $\left(L_{\text {radio }} \sim \mathcal{M}_{\mathrm{BH}}^{2.5}\right)$. This is what would be expected by AccretionDominated Accretion Flows (ADAF, see Abramowicz et al. 1995; Narayan \& Yi 1996) models. This correlation appears to hold over at least 3 order of magnitudes for $\mathcal{M}_{\mathrm{BH}}$ and, given its steepness, it was proposed as a tool to predict $\mathcal{M}_{\mathrm{BH}}$ from the simple observation of the radio flux. Additional support for a 


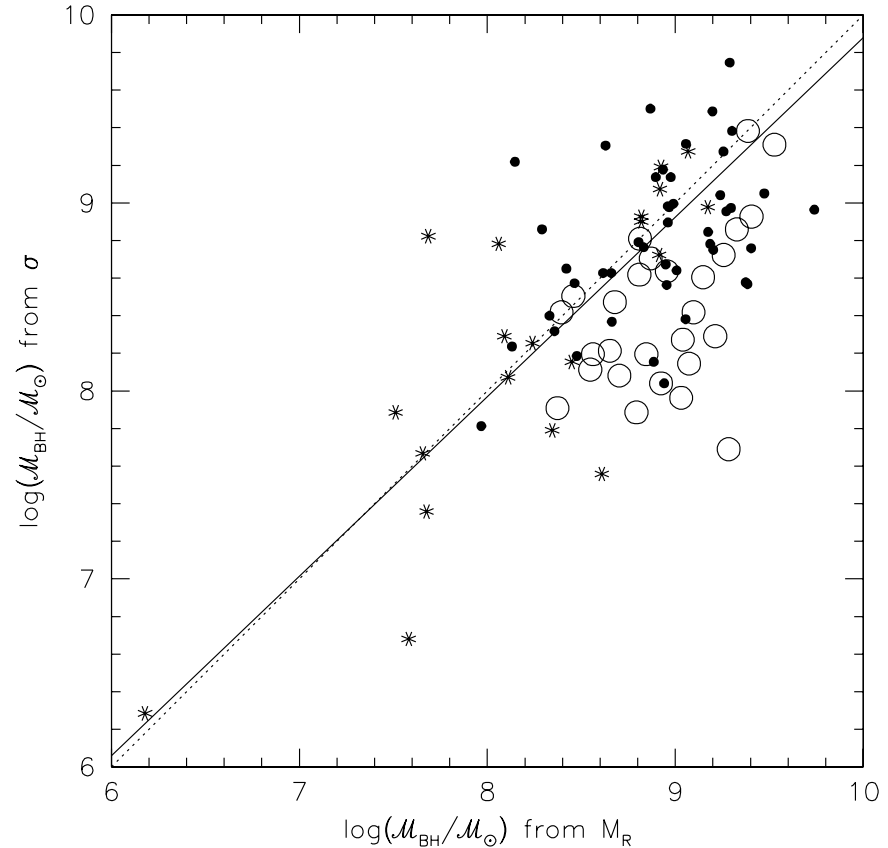

Fig. 2. The BH mass determinations obtained from $\mathcal{M}_{\mathrm{BH}}-M_{\mathrm{R}}$ and $\mathcal{M}_{\mathrm{BH}}-\log (\sigma)$ for the sample A of RG. A good agreement is found for the reduced sample A (filled circles), while the objects from SHI90 and FA89 (big open circles) exhibit a systematic deviation. For comparison we also plot $\mathcal{M}_{\mathrm{BH}}$ for the sample of 20 normal galaxies (asterisks). The solid line illustrates the best fit obtained for the reduced sample $\mathrm{A}: \log \left[\mathcal{M}_{\mathrm{BH}}(\sigma)\right]=0.28+0.94 \times \log \left[\mathcal{M}_{\mathrm{BH}}\left(M_{\mathrm{R}}\right)\right]$. The dotted line is the one-to-one relation.

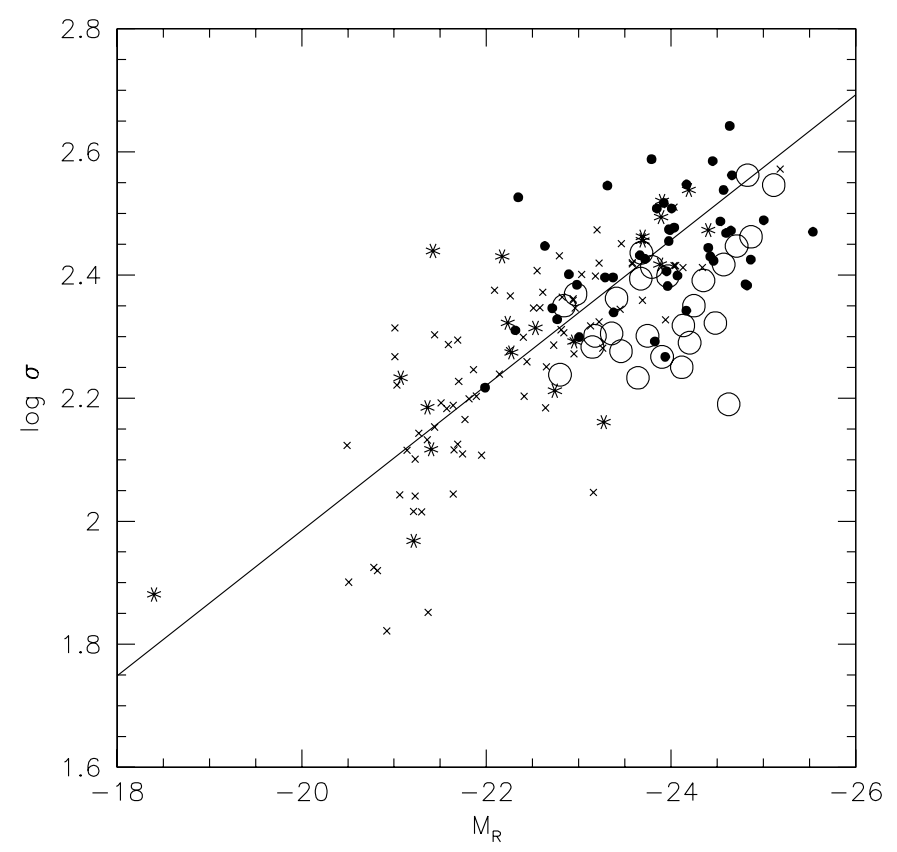

Fig. 3. Faber-Jackson relation for RGs in sample A and normal ellipticals. RGs from SHI90 and FA89 (open circles) have systematically lower $\sigma$ than the other RGs in the sample (filled circles). For comparison we also plot the 20 inactive Es (asterisks) and data for early-type galaxies from JFK96 (crosses). The solid line is our fit $\log \sigma=-0.375-0.118 \times M_{\mathrm{R}}$ to the data of reduced sample $\mathrm{A}$ and normal ellipticals in the JFK96 samples.

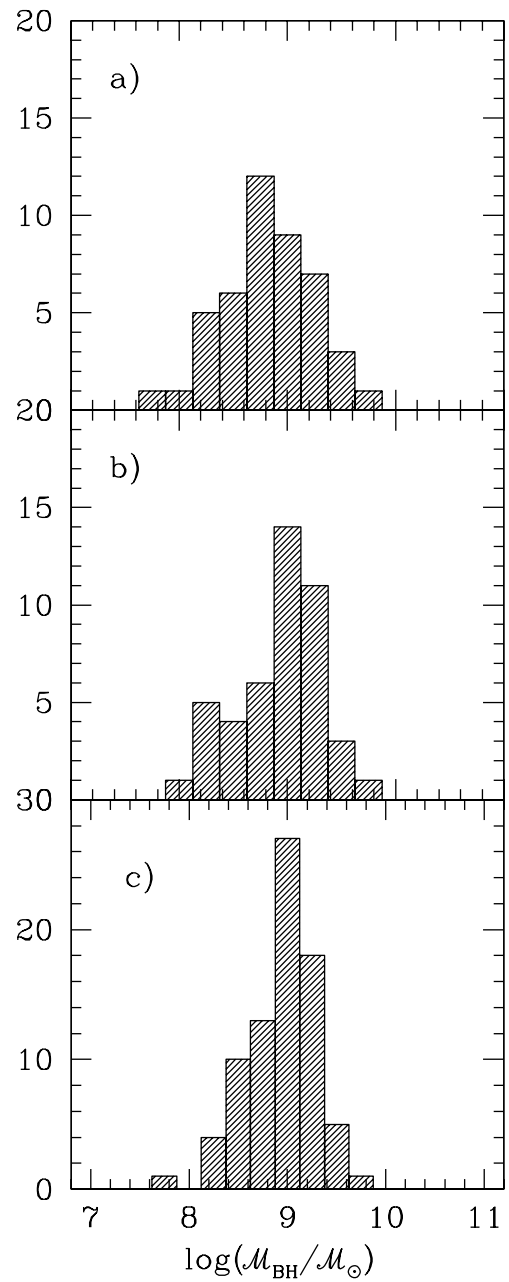

Fig. 4. a) The distribution of $\mathcal{M}_{\mathrm{BH}}$ for 46 low $z$ RGs derived from measurements of $\sigma$ (Eq. (2), reduced sample A); b)The distribution of $\mathcal{M}_{\mathrm{BH}}$ for 46 low $z$ RGs derived from measurements of the bulge luminosity (Eq. (1), reduced sample A); c) The distribution of $\mathcal{M}_{\mathrm{BH}}$ for 79 radiogalaxies derived from the bulge luminosity (Eq. (1), sample B).

link between $L_{\text {radio }}$ and $\mathcal{M}_{\mathrm{BH}}$ in various type of active galaxies was lead by Laor (2001) and Lacy et al. (2001).

However, a recent analysis by Ho (2002) of this relationship, for objects with a wide range of nuclear activity, argued that $\mathcal{M}_{\mathrm{BH}}$ is only loosely related to the radio power (both total and core emission). The poor correlation observed between the two quantities could therefore arise from indirect relations between radio luminosity, bulge mass and $\mathcal{M}_{\mathrm{BH}}$ and has no practical power to predict $\mathcal{M}_{\mathrm{BH}}$ from the radio luminosity.

We have investigated this issue using our samples of radiogalaxies for which radio power $\left(P_{\mathrm{tot}}\right)$ at $5 \mathrm{GHz}$ and $\mathcal{M}_{\mathrm{BH}}$ are available (Table 2). In Fig. 7 we show the data for our radio galaxies in the plane $\mathcal{M}_{\mathrm{BH}}-\log P_{5 \mathrm{GHz}}($ total). The two quantities are not correlated: Spearman correlation coefficient -0.174 , with significance of nonzero correlation 0.038 . The same result is found using the core radio power. Similarly, no significant correlation is found between the host galaxy mass $\mathcal{M}_{\text {bulge }}$ and the radio power, in agreement with the fact that $\mathcal{M}_{\mathrm{BH}}$ is tightly related to $\mathcal{M}_{\text {bulge }}$. 


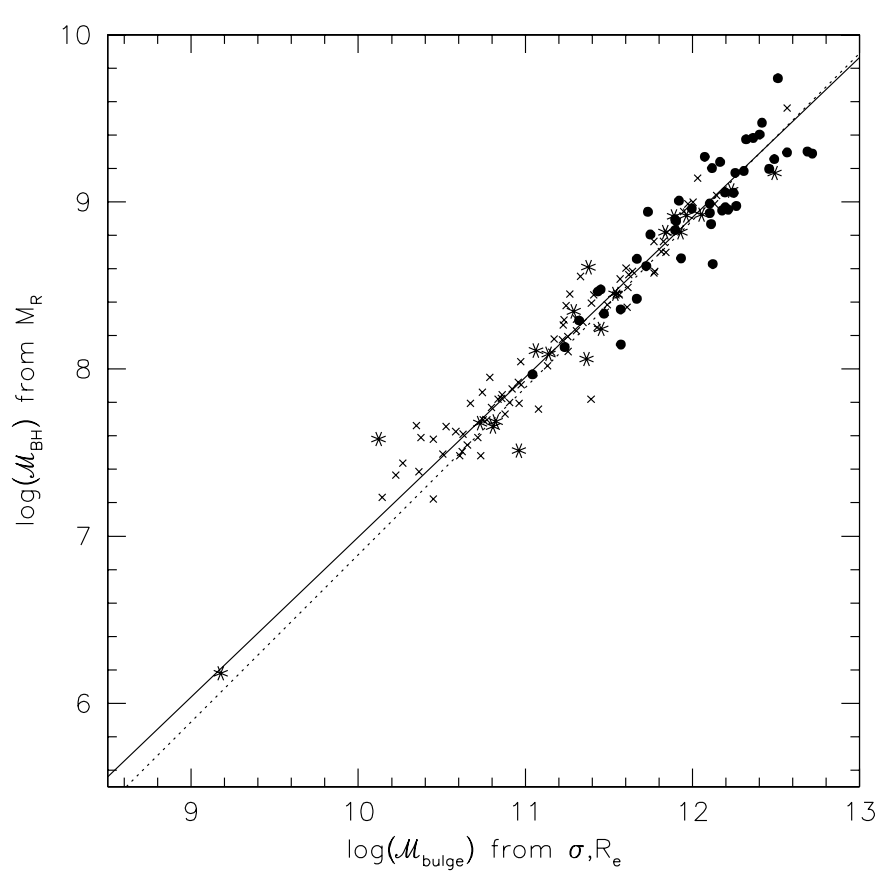

Fig. 5. The relation between $\mathcal{M}_{\mathrm{BH}}$ and $\mathcal{M}_{\text {bulge }}$ for RGs in the reduced sample A (filled circles), for the nearby inactive galaxies with BH mass determination (asterisks) and for JFK96 normal ellipticals (crosses). The solid line illustrates the fit obtained for galaxies in the reduced sample A and in JFK96 sample (see Eq. (3)). The dotted line represents the case of perfect linearity $(\eta=1)$ with $<\log \left(\mathcal{M}_{\mathrm{BH}} / \mathcal{M}_{\text {bulge }}\right)>=-3.11$.

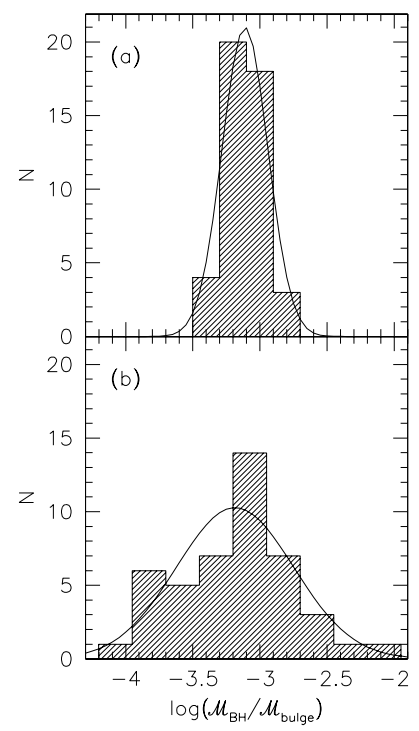

Fig. 6. The distribution of the ratio $\mathcal{M}_{\mathrm{BH}} / \mathcal{M}_{\mathrm{bulge}}$ for the reduced sample A of radiogalaxies: (a) $\mathcal{M}_{\mathrm{BH}}$ from Eq. (1) and $\mathcal{M}_{\text {bulge }}$ from $\sigma$ and $R_{\mathrm{e}}$; (b) $\mathcal{M}_{\mathrm{BH}}$ from Eq. (2) and $\mathcal{M}_{\text {bulge }}$ from $L_{\text {bulge }}$ assuming $\mathcal{M} / L \propto$ $L^{0.30}$. The solid lines represent Gaussian distributions with the same average values and rms of the corresponding dataset (see text).

In Fig. 8 we compare the distribution of our RGs over the plane $\mathcal{M}_{\mathrm{BH}}$ - radio power with that of other samples of galaxies at various levels of nuclear activity investigated by Ho (2002) and Oshlack et al. (2002). Note that the latter data refer to flat spectrum radio-sources whose flux is likely enhanced by

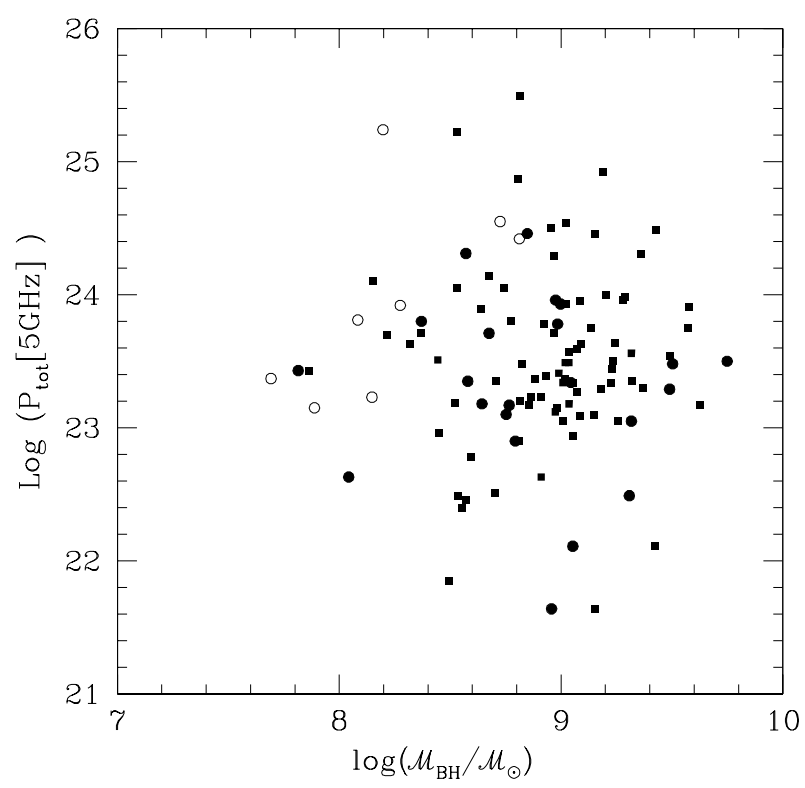

Fig. 7. $\mathcal{M}_{\mathrm{BH}}-\log P_{\text {tot }}(5 \mathrm{GHz})$ plane for RGs in sample A (filled circles, reduced sample A, open circles data from SHI90 and FA89) and sample B (filled squares). $\mathcal{M}_{\mathrm{BH}}$ is derived from $\sigma$ (see data in Tables 2 and 3). The two quantities are clearly not correlated.

Doppler boosting (beamed sources). Their observed luminosity therefore tends to occupy the higher region of the $P_{\text {tot }}-\mathcal{M}_{\mathrm{BH}}$ plane (see also Jarvis \& McLure 2002). Our data for RG are in agreement with the analysis by Ho (2002) and enforce his conclusion that radio power is poorly correlated with the mass of the central $\mathrm{BH}$ and therefore that the latter cannot practically be predicted by the measurement of the radio flux. If instead of the total radio luminosity we use the radio power of the core this picture remains basically unchanged. Together, the points in Fig. 8 appear to follow the trend of increasing $\mathcal{M}_{\mathrm{BH}}$ with higher radio luminosity. This behavior is consistent with the suggestion by Dunlop et al. (2002) that both active and inactive galaxies fall between two envelopes (following the $P_{\text {tot }} \propto \mathcal{M}_{\mathrm{BH}}^{2.5}$ law) in the $\mathcal{M}_{\mathrm{BH}}-P_{\text {tot }}$ plane. On the other hand the scatter of the points is rather large and do not produce a significant correlation of the two quantities. Our objects cover the topright part of the diagram where more massive $\mathrm{BH}$ and powerful radio sources are expected. Most of our points and those of Ho (2002) lie above the relation proposed by Franceschini et al. (1998) by $2-3$ order of magnitude. At low accretion rates ADAF models foresee that radio power depends on $\mathcal{M}_{\mathrm{BH}}$ as $P_{\mathrm{r}} \propto \mathcal{M}_{\mathrm{BH}}^{2.5}$. The location of points well above such relation may simply reflect the much higher level of activity (and likely of accretion).

\section{Summary and conclusions}

We used empirical relations between $\mathcal{M}_{\mathrm{BH}}$ and either the velocity dispersion $(\sigma)$ or the bulge luminosity $M_{\mathrm{R}}$ (bulge) derived for nearby early type galaxies to infer the mass of the central $\mathrm{BH}$ of low redshift radio galaxies.

The main conclusions of this study are:

1) Using only nearby galaxies of E-type morphology, for which $\mathrm{BH}$ masses are available, the two relationships $\mathcal{M}_{\mathrm{BH}}-\sigma$ 


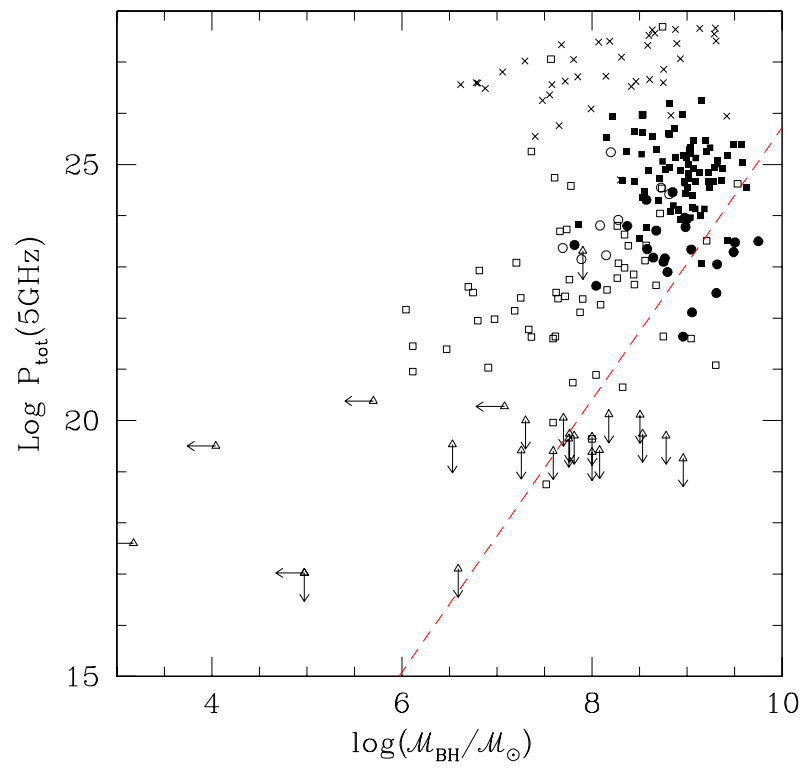

Fig. 8. $\mathcal{M}_{\mathrm{BH}}-\log P$ (total) relation for the samples A (filled circles, reduced sample A, open circles data from SHI90 and FA89) and B (filled squares) of radiogalaxies, compared with the objects (opens squares and triangles) studied by Ho (2002) and with radio loud quasars (crosses) investigated by Oshlack et al. (2002). The dashed line is the Franceschini et al. (1996) relation $\left(P_{\text {tot }} \propto \mathcal{M}_{\mathrm{BH}}^{2.5}\right)$.

and $\mathcal{M}_{\mathrm{BH}}-M_{\text {bulge }}$ exhibit a similar scatter $\left(\sim 0.4\right.$ in $\left.\log \mathcal{M}_{\mathrm{BH}}\right)$ which is also consistent with that expected from the estimated errors on the involved parameters.

2) We showed that when the dependence on the adopted cosmology (for the $\mathcal{M}_{\mathrm{BH}}-M_{\text {bulge }}$ relation) is properly taken into account, the two relations predict the same value of the central BH mass (within the expected uncertainties). This means that the $\mathrm{BH}$ mass can be reliably estimated from the observable parameter $M_{\mathrm{R}}$ (bulge) which is more easily measured than $\sigma$.

3) The inferred $\mathrm{BH}$ mass of low redshift radio galaxies is in the range $5 \times 10^{7}$ to $5 \times 10^{9} \mathcal{M}_{\odot}$.

4) We found that the central BH mass is linearly correlated with that of its host galaxy; the average Black Hole to bulge mass ratio is $<\log \left[\mathcal{M}_{\mathrm{BH}} / \mathcal{M}_{\text {bulge }}\right]>\sim-3.1$.

5) The total (or core) radio power at $5 \mathrm{GHz}$ is not correlated either with the mass of the central $\mathrm{BH}$ nor with that of the galaxy; the radio power is always in excess by $2-3$ order of magnitude with respect to what would be expected from low accretion rate models (ADAF).

Acknowledgements. We wish to acknowledge the anonymous referee for valuable comments which lead to the improvement of this paper. This work has received partial support under contracts COIN2001/028773-004, ASI-IR-35 and ASI-IR-73-01.

\section{References}

Adams, F. C., Graff, D. S., \& Richstone, D. O. 2001, ApJ, 551, L31 Abramowicz, M. A., \& Lasota, J. P. 1995, Comm. Astrophys. 18, 141 Becker, R. H., White, R. L., \& Edwards, A. L. 1991, ApJS, 75, 1 Bender, R., Burstein, D., \& Faber, S. M. 1992, ApJ, 399, 462
Bettoni, D., Falomo, R., Fasano, G., et al. 2001, A\&A, 380, 471

Bressan, A., Chiosi, C., \& Fagotto, F. 1994, ApJS, 94, 63

Colina, L., \& de Juan, L. 1995, ApJ, 448, 548

Dufour, R., Harvel, C., Martins, D., et al. 1979, AJ, 84, 284

Dunlop, J. S., McLure, R. J., Kukula, M. J., et al. 2002 [astro-ph/0108397]

Ekers, R. D., Wall, J. V., Shaver, P. A., et al. 1989, MNRAS, 236, 737

Faber, S., Tremaine, S., Ajhar, E. A., et al. 1997, AJ, 114, 1771

Faber, S. M., Wegner, G., Burstein, D., et al. 1989, ApJS, 69, 763(FA89)

Falomo, R., Carangelo, N., \& Treves, A. 2002, MNRAS, submitted

Fasano, G., \& Vio, R. 1988, Bull. Inf. CDS No. 35, 191

Fasano, G., Falomo, R., \& Scarpa, R. 1996, MNRAS, 282, 40

Ferrarese, L. 2002 [astro-ph/0207050]

Ferrarese, L., \& Merritt, D. 2000, ApJ, 539, L9

Franceschini, A., Vercellone, S., \& Fabian, A. C. 1998, MNRAS, 297, 817

Fukugita, M., Shimasaku, K., \& Ichikawa, T. 1995, PASP, 107, 945

Gebhardt, K., Bender, R., Bower, G., et al. 2000, ApJ, 539, L13

Gonzalez-Serrano, J. I., Carballo, R., \& Perez-Fournon, J. 1993, AJ, 105,1710

Gonzalez-Serrano, J. I., \& Carballo, R. 2000, A\&A, 142, 353

Govoni, F., Falomo, R., Fasano, G., \& Scarpa, R. 2000a, A\&A, 353, 507

Govoni, F., Falomo, R., Fasano, G., \& Scarpa, R. 2000b, A\&AS, 143, 369

Griffith, M. R., Wright, A. E., Burke, B. F., \& Ekers, R. D. 1994, ApJS, 90, 179

Griffith, M. R., Wright, A. E., Burke, B. F., \& Ekers, R. D. 1995, ApJS, 97, 347

Haehlnet, M. G., \& Kauffmann, G. 2002, MNRAS, 318, L35

Ho, L. C. 2002, ApJ, 564, 120

Jarvis, M. J., \& McLure, R. J. 2002 [astro-ph/0208390]

Jørgensen, I., Franx, M., \& Kjærgaard, P. 1996, MNRAS, 280, 167(JFK96)

Kormendy, J., \& Gebhardt, K. 2001, 20th Texas Symp. on relativistic astrophisycs, ed. J. C. Wheeler, \& H. Martel, AIP Conf. Proc., 586,363

Küehr, H., Witzel, A., Pauliny-Thoth, I. I. K., \& Nauber, U. 1981, A\&AS, 45, 367

Lacy, M., Laurent-Muehleisen, S. A., Ridgway, S. E., Becker, R. H., \& White, R. L. 2001, ApJ, 551, L17

Laor, A. 2001, ApJ, 553, 677

Ledlow, M. J., \& Owen, F. N. 1995, AJ, 110, 1959

Merritt, D., \& Ferrarese, L. 2001, MNRAS, 320, L30

Magorrian, J., Tremaine, S., Richstone, D., et al. 1998, AJ, 115, 2285

McLure, R. J., \& Dunlop, J. S. 2002, MNRAS, 331, 795

Narayan, R., \& Yi, I. 1996, ApJ, 444, 231

Oshlack, A., Webster, R., \& Whiting, M. 2002, ApJ, 576, 81

Owen, F. N., \& Laing, R. A. 1989, MNRAS, 238, 357

Prugniel, Ph., \& Maubon, G. 2000, Dynamics of Galaxies: from the Early Universe to the Present, ASP Conf. Ser., 197, 403

Silk, J., \& Rees, M. J. 1998, A\&A, 331, L4

Smith, E. P., \& Heckman, T. M. 1989, ApJ, 341, 658

Smith, E. P., Heckman, T. M., \& Illingworth, G. D. 1990, ApJ, 356, 399(SHI90)

Stark, A. A., Gammie, C. F., Wilson, R. W., et al. 1992, ApJS, 79, 77

Tonry, J. L., Dressler, A., Blakeslee, J. P., et al. 2001, ApJ, 546, 681

Tremaine, S., Gebhardt, K., Bender, R., et al. 2002, ApJ, 574, 740

de Vaucouleurs, G., de Vaucouleurs, A., Corwin, H. G., et al. 1991, Third Reference Catalogue of Bright Galaxies (RC3) (New York: Springer-Verlag)

Wall, J. V., \& Peacock, J. A. 1985, MNRAS, 216, 173 (WP) 